\title{
Bifurcation Analysis of a Ratio-Dependent Holling- Tanner Model with Cross-Diffusion
}

\author{
Zhen $\mathrm{Li}^{1}$, Yan Meng ${ }^{1, *}$ and Yongan $\mathrm{Ye}^{2}$ \\ ${ }^{1}$ School of Mathematics and Physics, University of Science and Technology Beijing, Beijing, 100083, PR China \\ ${ }^{2}$ Dongzhimen Hospital Beijing University of Chinese Medicine, Beijing, 100083, PR China \\ ${ }^{*}$ Corresponding author
}

\begin{abstract}
In this paper, we investigate a discrete space-time predator-prey model with ratio-dependent function response. The model is given by a coupled map lattice framework, it takes a nonlinear relationship between predator-prey reaction stage and dispersal stage. Through stability analysis and bifurcation analysis, the parameter conditions of Hopf bifurcation is obtained. When adding the cross-diffusion, we obtain the parameter conditions of Turing instability. Numerical simulations verify the theoretical analysis and show a series of spatial patterns.
\end{abstract}

Keywords—discrete model; hopf bifurcation; turing instability; pattern formation

\section{INTRODUCTION}

The predator-prey system is one of the basic ecological models widely existing in nature. Since Lotka and Volterra discovered the basic model of predator-prey systems, more and more researchers investigate the dynamical behaviors of predator-prey system [1-4]. In recent decades, dynamical behaviors become more and more abundant and complex. The spatial pattern formation of predator-prey systems is an important issue in biological science. Since Alan Turing put forward the reaction diffusion equations of chemical and found that diffusion could destabilize the stable equilibrium [6]. Currently, reaction-diffusion theory is gradually maturing and is applied into many fields [5,7].

Many researchers have studied time- and space-continuous predator-prey systems. But the discrete model is more suitable for the study of biological system, such as scattered habitats, biological population of non-overlapping. Comparing with the continuous dynamical models, the discrete models show more accuracy in describing population dynamics and exhibit many complex nonlinear characteristics [8].

In this paper, we focus on the discrete form of HollingTanner model with cross diffusion. Holling-Tanner model is one of the most famous predator-prey models and its functional response is Holling Type II $[9,10]$. In order to describe some other situations observed in the fields, this paper introduce a ratio-dependent predator-prey model.

The outline of this paper is as follows. In section II, we give the discrete space-time predator-prey model. In section III, we discuss that the stability of equilibrium points and the parameter conditions of the Hopf bifurcation and Turing instability. In section IV, numerical simulations are given to verify the theoretical results. Concluding remarks are given in section $\mathrm{V}$.

\section{The Discrete SPACE-TIME PredATOR-PREy MODEL}

In this section, we begin to study the Holling-Tanner model with a ratio-dependent type [11], can be described by the following reaction-diffusion equations:

$$
\left\{\begin{array}{l}
\frac{\partial N}{\partial T}=r N\left(1-\frac{N}{k}\right)-\frac{m N P}{N+a P}+D_{11} \nabla^{2} N+D_{12} \nabla^{2} P \\
\frac{\partial P}{\partial T}=\operatorname{Ps}\left(1-\frac{h P}{N}\right)+D_{21} \nabla^{2} N+D_{22} \nabla^{2} P
\end{array}\right.
$$

where $\mathrm{N}$ and $\mathrm{P}$ represent the biomass of prey and predator population, $\mathrm{T}$ denotes time; $\mathrm{r}, \mathrm{k}, \mathrm{m}, \mathrm{a}, \mathrm{s}, \mathrm{h}$ are positive which $\mathrm{r}$ is the intrinsic growth rate, $\mathrm{k}$ is the carrying capacity of the prey, $m$ is the predation rate or capturing rate of prey by predator, $a$ is the interference coefficient of the predator, $s$ is the predator intrinsic growth rate and $h$ is the conversion rate of prey into predator biomass; $\nabla^{2} N$ and $\nabla^{2} P$ express the diffusion of the prey and the predator in space, $D_{11}$ and $D_{22}$ are the selfdiffusion coefficients, $D_{12}$ and $D_{21}$ are the cross-diffusion coefficients of predator and prey; $\nabla^{2}=\partial / \partial x^{2}+\partial / \partial y^{2}$ is the usual Laplace operator in two-dimensional space, $\mathrm{x}$ and $\mathrm{y}$ are the space coordinates of $\mathrm{N}$ and $\mathrm{P}$.

Using the scaling $n=\frac{N}{k}, p=\frac{m P}{r k}, t=r T, \alpha=\frac{a r}{m}, \delta=\frac{s}{r}, \beta=\frac{h r}{m}$

We get the following equation

$$
\left\{\begin{array}{l}
\frac{\partial n}{\partial t}=n(1-n)-\frac{n p}{n+\alpha p}+d_{11} \nabla^{2} n+d_{12} \nabla^{2} p \\
\frac{\partial p}{\partial t}=\delta p\left(1-\frac{\beta p}{n}\right)+d_{21} \nabla^{2} n+d_{22} \nabla^{2} p
\end{array}\right.
$$

where $d_{11}=\frac{k D_{11}}{r}, d_{12}=\frac{r k D_{12}}{m^{2}}, d_{21}=\frac{k m D_{21}}{r^{2}}, d_{22}=\frac{k D_{22}}{m}$.

The classical method for developing the discrete space-time predator-prey model is applying Euler discretization on (2), we set a time interval of $\tau$ and space interval $h$ for discretizing (2), and a rectangular domain including $n \times n$ lattices. Each lattice stands for a spatial spot, is ascribed to prey biomass and predator biomass. Accordingly, two state variables $n_{(i, j, t)}$ and 
$p_{(i, j, t)}\left(i, j, t \in Z^{+}\right.$and $\left.1 \leq i, j \leq n\right)$ are defined, which represent the prey density and predator density in lattice $(i, j)$ at time t.

$$
\begin{aligned}
& n_{(i, j, t+1)}=f\left(n_{(i, j, t)}, p_{(i, j, t)}\right)+\frac{\tau}{h^{2}} d_{11} \nabla_{d}^{2} n_{(i, j, t)}+\frac{\tau}{h^{2}} d_{12} \nabla_{d}^{2} p_{(i, j, t)}, \\
& p_{(i, j, t+1)}=g\left(n_{(i, j, t)}, p_{(i, j, t)}\right)+\frac{\tau}{h^{2}} d_{21} \nabla_{d}^{2} n_{(i, j, t)}+\frac{\tau}{h^{2}} d_{22} \nabla_{d}^{2} p_{(i, j, t)}
\end{aligned}
$$

where

$$
\begin{aligned}
& f(n, p)=n+\tau n(1-n)-\frac{\tau n p}{n+\alpha p}, \\
& g(n, p)=p+\tau \delta p\left(1-\frac{\beta p}{n}\right) .
\end{aligned}
$$

and $\nabla_{d}^{2}$ is the discrete form of Laplace operator, and shown by the following equation

$$
\begin{aligned}
& \nabla_{d}^{2} n_{(i, j, t)}=n_{(i-1, j, t)}+n_{(i+1, j, t)}+n_{(i, j-1, t)}+n_{(i, j+1, t)}-4 n_{(i, j, t)}, \\
& \nabla_{d}^{2} p_{(i, j, t)}=p_{(i-1, j, t)}+p_{(i+1, j, t)}+p_{(i, j-1, t)}+p_{(i, j+1, t)}-4 p_{(i, j, t)} .
\end{aligned}
$$

In the coupled map lattice framework, the dynamics at each step iteration of the model consists of a reaction stage and a dispersal stage. The dispersal stage is obtained by discretizing the spatial term of (2), i.e.,

$$
\begin{aligned}
& n_{(i, j, t)}^{\prime}=n_{(i, j, t)}+\frac{\tau}{h^{2}} d_{11} \nabla_{d}^{2} n_{(i, j, t)}+\frac{\tau}{h^{2}} d_{12} \nabla_{d}^{2} p_{(i, j, t)}, \\
& p_{(i, j, t)}^{\prime}=p_{(i, j, t)}+\frac{\tau}{h^{2}} d_{21} \nabla_{d}^{2} n_{(i, j, t)}+\frac{\tau}{h^{2}} d_{22} \nabla_{d}{ }^{2} p_{(i, j, t)} .
\end{aligned}
$$

The reaction stage can be expressed as

$$
\begin{aligned}
& n_{(i, j, t+1)}=f\left(n_{(i, j, t)}^{\prime}, p_{(i, j, t)}^{\prime}\right), \\
& p_{(i, j, t+1)}=g\left(n_{(i, j, t)}^{\prime}, p_{(i, j, t)}^{\prime}\right) .
\end{aligned}
$$

(6) and (7) describe a discrete space-time predator-prey system with self- and cross-diffusion. In the model, all the parameters should be the positive and values of $n_{(i, j, t)}$ and $p_{(i, j, t)}$ are non-negative. We notice that the above discrete model cannot describe the dynamics on the boundary. For applying the discrete model, we set the periodic boundary conditions as follows:

$$
\begin{aligned}
& n_{(i, 1, t)}=n_{(i, n+1, t)}, n_{(i, 0, t)}=n_{(i, n, t)}, n_{(1, j, t)}=n_{(n+1, j, t)}, n_{(0, j, t)}=n_{(n, j, t)} . \\
& p_{(i, 1, t)}=p_{(i, n+1, t)}, p_{(i, 0, t)}=p_{(i, n, t)}, p_{(1, j, t)}=p_{(n+1, j, t)}, p_{(0, j, t)}=p_{(n, j, t)} .
\end{aligned}
$$

\section{CONDITIONS FOR PATTERN FORMATION}

Next, the discrete model is employed to investigate the special pattern formation of predator and prey, and the conditions for pattern formation should be determined.

\section{A. Conditions for Stable Homogeneous Stationary State}

The homogeneous stationary states of the discrete model require

$$
\begin{aligned}
\nabla_{d}^{2} n_{(i, j, t)} & \equiv 0, \\
\nabla_{d}^{2} p_{(i, j, t)} & \equiv 0 .
\end{aligned}
$$

for all of $i$ and $j$. Here, the equation of discrete model becomes the following form

$$
\left\{\begin{array}{l}
n_{(i, j, t+1)}=n_{(i, j, t)}+\tau\left(n_{(i, j, t)}\left(1-n_{(i, j, t)}\right)-\frac{n_{(i, j, t)} p_{(i, j, t)}}{n_{(i, j, t)}+\alpha p_{(i, j, t)}}\right), \\
p_{(i, j, t+1)}=p_{(i, j, t)}+\tau\left(\delta p_{(i, j, t)}\left(1-\frac{\beta p_{(i, j, t)}}{n_{(i, j, t)}}\right)\right) .
\end{array}\right.
$$

The equilibrium point is calculated as

$$
\begin{aligned}
& \left(n_{0}, p_{0}\right)=(1,0) \\
& \left(n_{1}, p_{1}\right)=\left(1-\frac{1}{\alpha+\beta}, \frac{1}{\beta}-\frac{1}{\beta^{2}+\alpha \beta}\right)
\end{aligned}
$$

Jacobi matrix is

$$
J(n, p)=\left(\begin{array}{cc}
\lambda-1-\tau\left(1-2 n-\frac{\alpha p^{2}}{(n+\alpha p)^{2}}\right) & \frac{\tau n^{2}}{(n+\alpha p)^{2}} \\
-\frac{\tau \delta \beta p^{2}}{n^{2}} & \lambda-1-\tau\left(\delta-2 \delta \beta \frac{p}{n}\right)
\end{array}\right)
$$

Substituting the values of $\left(n_{0}, p_{0}\right)$ and $\left(n_{1}, p_{1}\right)$ into (11) and calculating its two eigenvalues $\lambda_{1}$ and $\lambda_{2}$ of the Jacobi matrix. According to the research of [12], the stability criterion can be obtained as follows: if $\left|\lambda_{1}\right|<1$ and $\left|\lambda_{2}\right|<1$, then the corresponding fixed point is stable; if $\left|\lambda_{1}\right|<1$ and $\left|\lambda_{2}\right|>1$ or $\left|\lambda_{1}\right|>1$ and $\left|\lambda_{2}\right|<1$, then the corresponding fixed point is a saddle point, which is also unstable; if $\left|\lambda_{1}\right|>1$ and $\left|\lambda_{2}\right|>1$, the corresponding fixed point is unstable.

For fixed point $\left(n_{0}, p_{0}\right)$, we have

$$
J\left(n_{0}, p_{0}\right)=\left(\begin{array}{cc}
\lambda-1+\tau & 1 \\
0 & \lambda-1-\tau \delta
\end{array}\right)
$$


From the above criteria, when $0<\tau<2$ and $-2<\tau \delta<0$, $\left(n_{0}, p_{0}\right)$ is stable. And the system is stable at $\left(n_{1}, p_{1}\right)$, each parameter should satisfies the following conditions:

$$
\begin{aligned}
& a_{11}+a_{22}<1+a_{11} a_{22}-a_{12} a_{21}, \\
& a_{11}+a_{22}>-\left(1+a_{11} a_{22}-a_{12} a_{21}\right), \\
& a_{11} a_{22}-a_{12} a_{21}<1 .
\end{aligned}
$$

where

$$
\begin{aligned}
& a_{11}=1+\tau\left(1-2 n_{1}\right)-\frac{\tau \alpha p_{1}^{2}}{\left(n_{1}+\alpha p_{1}\right)^{2}}, \quad a_{12}=-\frac{\tau n_{1}^{2}}{\left(n_{1}+\alpha p_{1}\right)^{2}}, \\
& a_{21}=\tau \delta \beta\left(\frac{p_{1}}{n_{1}}\right)^{2}, \quad a_{22}=1+\tau \delta-\frac{2 \tau \delta \beta p_{1}}{n_{1}} .
\end{aligned}
$$

\section{B. Conditions for Hopf Bifurcation}

According to the discrete system bifurcation theory, the Hopf bifurcation is independent in space, so next we will not consider the diffusion term in the study of Hopf bifurcation, i.e., $\nabla_{d}^{2} n_{(i, j, t)}=0$ and $\nabla_{d}^{2} p_{(i, j, t)} \equiv 0$.

The eigenvalues of Jacobi matrix of $\left(n_{1}, p_{1}\right)$ are as follows

$$
\lambda_{1,2}=\frac{1}{2}\left(a_{11}+a_{22} \pm \sqrt{\left(a_{11}-a_{22}\right)^{2}+4 a_{12} a_{21}}\right)
$$

The first condition for the appearance of the Hopf bifurcation is that $\lambda_{1}$ and $\lambda_{2}$ is a pair of complex number with modulus one, i.e.

$$
\left(a_{11}-a_{22}\right)^{2}+4 a_{12} a_{21}<0
$$

When $a_{11} a_{22}-a_{12} a_{21}=1$, i.e.

$$
\tau=\tau_{0}=-\frac{\left(1-2 n_{1}\right)+\delta-\frac{2 \delta \beta p_{1}}{n_{1}}-\frac{\alpha p_{1}^{2}}{\left(n_{1}+\alpha p_{1}\right)^{2}}}{\delta\left(1-2 n_{1}\right)-2 \frac{\delta \beta p_{1}\left(1-2 n_{1}\right)}{n_{1}}+\frac{(\beta-\alpha) \delta p_{1}^{2}}{\left(n_{1}+\alpha p_{1}\right)^{2}}+\frac{2 \alpha \beta \delta p_{1}^{3}}{n_{1}\left(n_{1}+\alpha p_{1}\right)^{2}}}
$$

We have $\left|\lambda_{1}\right|=1$ and $\left|\lambda_{2}\right|=1$.

Next, we change the $\left(n_{1}, p_{1}\right)$ to the origin by the following transformation

$$
\begin{aligned}
& u=n-n_{1} \\
& v=p-p_{1}
\end{aligned}
$$

The following mapping is obtained

$$
\left(\begin{array}{l}
u \\
v
\end{array}\right) \rightarrow\left(\begin{array}{c}
a_{11} u+a_{12} v+\frac{a_{13}}{2} u^{2}+a_{14} u v+\frac{a_{15}}{2} v^{2}+\frac{a_{16}}{6} u^{3}+\frac{a_{17}}{2} u^{2} v+\frac{a_{18}}{2} u v^{2}+\frac{a_{19}}{6} v^{3}+O\left((|u|+|v|)^{4}\right) \\
a_{21} u+a_{22} v+\frac{a_{33}}{2} u^{2}+a_{24} u v+\frac{a_{25}}{2} v^{2}+\frac{a_{26}}{6} u^{3}+\frac{a_{27}}{2} u^{2} v+\frac{a_{38}}{2} u v^{2}+\frac{a_{29}}{6} v^{3}+O\left((|u|+\mid v)^{4}\right)
\end{array}\right)
$$

where $O\left((|u|+|v|)^{4}\right)$ describes a function with order at least four in the variables $(u, v)$, and

$$
\begin{aligned}
& a_{13}=-2 \tau+\frac{\tau n_{1} p_{1}+2 \tau \alpha\left(p_{1}\right)^{2}}{\left(n_{1}+\alpha p_{1}\right)^{3}}, a_{14}=-\frac{2 \tau \alpha n_{1} p_{1}}{\left(n_{1}+\alpha p_{1}\right)^{3}}, a_{15}=\frac{2 \tau \alpha\left(n_{1}\right)^{2}}{\left(n_{1}+\alpha p_{1}\right)^{3}}, \\
& a_{16}=-\frac{6 \tau \alpha\left(p_{1}\right)^{2}}{\left(n_{1}+\alpha p_{1}\right)^{4}}, a_{17}=\frac{-2 \tau \alpha^{2}\left(p_{1}\right)^{2}+4 \tau \alpha n_{1} p_{1}}{\left(n_{1}+\alpha p_{1}\right)^{4}}, \\
& a_{18}=\frac{-2 \tau \alpha\left(n_{1}\right)^{2}+4 \tau \alpha^{2} n_{1} p_{1}}{\left(n_{1}+\alpha p_{1}\right)^{4}}, a_{19}=-\frac{6 \tau \alpha^{2}\left(n_{1}\right)^{2}}{\left(n_{1}+\alpha p_{1}\right)^{4}}, \\
& a_{23}=-\frac{2 \tau \delta \beta}{\left(n_{1}\right)^{3}}, a_{24}=\frac{2 \tau \delta \beta p_{1}}{\left(n_{1}\right)^{2}}, a_{25}=-\frac{2 \tau \delta \beta}{n_{1}}, a_{26}=-\frac{6 \tau \delta \beta}{\left(n_{1}\right)^{4}}, \\
& a_{27}=-\frac{4 \tau \delta \beta p_{1}}{\left(n_{1}\right)^{3}}, a_{28}=\frac{2 \tau \delta \beta}{\left(n_{1}\right)^{2}}, a_{29}=0 .
\end{aligned}
$$

In order to facilitate subsequent discussions, the two eigenvalue values can be written in the following form

$$
\lambda\left(\tau_{0}\right), \bar{\lambda}\left(\tau_{0}\right)=-\frac{p\left(\tau_{0}\right)}{2} \pm \frac{i}{2} \sqrt{4 q\left(\tau_{0}\right)-p^{2}\left(\tau_{0}\right)}=\alpha_{1} \pm i \alpha_{2}
$$

where

$$
\begin{aligned}
p\left(\tau_{0}\right)= & -2-\tau_{0}\left(1-2 n_{1}-\frac{\alpha p_{1}^{2}}{\left(n_{1}+\alpha p_{1}\right)^{2}}+\delta-2 \delta \beta \frac{p_{1}}{n_{1}}\right), \\
q\left(\tau_{0}\right)= & 1+\tau_{0}\left(1-2 n_{1}-\frac{\alpha p_{1}^{2}}{\left(n_{1}+\alpha p_{1}\right)^{2}}+\delta-2 \delta \beta \frac{p_{1}}{n_{1}}\right) \\
& +\tau_{0}^{2}\left(\left(1-2 n_{1}-\frac{\alpha p_{1}^{2}}{\left(n_{1}+\alpha p_{1}\right)^{2}}\right)\left(\delta-2 \delta \beta \frac{p_{1}}{n_{1}}\right)+\frac{\delta \beta p_{1}^{2}}{\left(n_{1}+\alpha p_{1}\right)^{2}}\right) .
\end{aligned}
$$

We can know from the above that $|\lambda|=\sqrt{q\left(\tau_{0}\right)}=1$.

Another condition of the existence of Hopf bifurcation is

$$
d=\left.\frac{d|\lambda(\tau)|}{d \tau}\right|_{\tau=\tau_{0}}=-\frac{1}{2}\left(1-2 n_{1}+\delta-\frac{2 \delta \beta p_{1}}{n_{1}}-\frac{\alpha p_{1}^{2}}{\left(n_{1}+\alpha p_{1}\right)^{2}}\right) \neq 0 .
$$

In addition, the following conditions are also required

$$
\left(\lambda\left(\tau_{0}\right)\right)^{\theta} \neq 1, \theta \neq 1,2,3,4
$$

which is equivalent to

$$
p\left(\tau_{0}\right) \neq-2,0,1,2
$$


It is known by (21), $p^{2}\left(\tau_{0}\right) \neq 4$. Hence, $p\left(\tau_{0}\right) \neq-2,2$, then the requirement of $p\left(\tau_{0}\right) \neq 0,1$ lead to

$$
\tau_{0}\left(1-2 n_{1}-\frac{\alpha p_{1}}{\left(n_{1}+\alpha p_{1}\right)^{2}}+\delta-2 \delta \beta \frac{p_{1}}{n_{1}}\right) \neq-2,-3
$$

Next, we study the normal form of (19) when $\tau=\tau_{0}$.

Applying the transformation

$$
\left(\begin{array}{l}
u \\
v
\end{array}\right)=\left(\begin{array}{cc}
a_{12} & 0 \\
\alpha_{1}-a_{11} & -\alpha_{2}
\end{array}\right)\left(\begin{array}{l}
U \\
V
\end{array}\right)
$$

Then

$$
\left(\begin{array}{l}
U \\
V
\end{array}\right) \rightarrow\left(\begin{array}{cc}
\alpha_{1} & -\alpha_{2} \\
\alpha_{2} & \alpha_{1}
\end{array}\right)\left(\begin{array}{l}
U \\
V
\end{array}\right)+\frac{1}{a_{12} \alpha_{2}}\left(\begin{array}{l}
F_{1}(U, V) \\
G_{1}(U, V)
\end{array}\right)
$$

where

$$
\begin{aligned}
F_{1}(U, V)= & \frac{a_{13} \alpha_{2}}{2} u^{2}+a_{14} \alpha_{2} u v+\frac{a_{15} \alpha_{2}}{2} v^{2}+\frac{a_{16} \alpha_{2}}{6} u^{3}+\frac{a_{17} \alpha_{2}}{2} u^{2} v \\
& +\frac{a_{18} \alpha_{2}}{2} u v^{2}+\frac{a_{19} \alpha_{2}}{6} v^{3}+O\left((|u|+|v|)^{4}\right), \\
G_{1}(U, V)= & \left(\frac{a_{13}\left(\alpha_{1}-a_{11}\right)}{2}-\frac{a_{12} a_{23}}{2}\right) u^{2}+\left(a_{14}\left(\alpha_{1}-a_{11}\right)-a_{12} a_{24}\right) u v \\
& +\left(\frac{a_{15}\left(\alpha_{1}-a_{11}\right)}{2}-\frac{a_{12} a_{25}}{2}\right) v^{2}+\left(\frac{a_{16}\left(\alpha_{1}-a_{11}\right)}{6}-\frac{a_{12} a_{26}}{6}\right) u^{3} \\
& +\left(\frac{a_{17}\left(\alpha_{1}-a_{11}\right)}{2}-\frac{a_{12} a_{27}}{2}\right) u^{2} v+\left(\frac{a_{18}\left(\alpha_{1}-a_{11}\right)}{2}-\frac{a_{12} a_{28}}{2}\right) u v^{2} \\
& +\left(\frac{a_{19}\left(\alpha_{1}-a_{11}\right)}{6}-\frac{a_{12} a_{29}}{6}\right) v^{3}+O\left((|u|+|v|)^{4}\right) .
\end{aligned}
$$

In order to promise the appearance of the Hopf bifurcation, the following $a$ must not be zero.

$$
a=-\operatorname{Re}\left(\frac{(1-2 \bar{\lambda}) \bar{\lambda}^{2}}{1-\lambda} \xi_{11} \xi_{20}\right)-\frac{1}{2}\left|\xi_{11}\right|^{2}-\left|\xi_{02}\right|^{2}+\operatorname{Re}\left(\bar{\lambda} \xi_{21}\right) \neq 0 .
$$

where

$$
\begin{aligned}
& \xi_{20}=\frac{1}{8}\left(\left(F_{U U}-F_{W}+2 G_{U V}\right)+i\left(G_{U U}-G_{W}-2 F_{U V}\right)\right), \\
& \xi_{11}=\frac{1}{4}\left(\left(F_{U U}+F_{W}\right)+i\left(G_{U U}+G_{W}\right)\right), \\
& \xi_{02}=\frac{1}{8}\left(\left(F_{U U}-F_{W}-2 G_{U V}\right)+i\left(G_{U U}-G_{W}+2 F_{U V}\right)\right), \\
& \xi_{21}=\frac{1}{16}\left(\left(F_{U U U}+F_{U W}+G_{U U V}+G_{U V}\right)+i\left(G_{U U U}+G_{U W}-F_{U U V}-F_{U V}\right)\right) .
\end{aligned}
$$

In which $F=\frac{F_{1}}{a_{12} \alpha_{2}}, G=\frac{G_{1}}{a_{12} \alpha_{2}}, F_{U U}=\left.\frac{\partial^{2} F}{\partial U^{2}}\right|_{U=V=0}, F_{U U U}=\left.\frac{\partial^{3} F}{\partial U^{3}}\right|_{U=V=0}$. and likewise for all other similar terms.

\section{Conditions for Turing Bifurcation}

Turing instability analysis is performed at the nontrivial homogeneous stationary state $\left(n_{1}, p_{1}\right)$. In the analysis of the Turing bifurcation, $\nabla_{d}^{2} n_{(i, j, t)}$ and $\nabla_{d}^{2} p_{(i, j, t)}$ are not always be zero, the eigenvalues of the operator $\nabla_{d}^{2}$ are determined firstly.

Next, considering the following equation

$$
\nabla_{d}^{2} X^{i j}+\lambda X^{i j}=0
$$

And the boundary conditions are as follows

$$
X^{i, 1}=X^{i, n+1}, X^{i, 0}=X^{i, n}, X^{1, j}=X^{n+1, j}, X^{0, j}=X^{n, j} .
$$

Applying the methods described in the eigenvalues of the Laplace operator can be formulated as

$$
\lambda_{k l}=4\left(\sin ^{2}\left(\frac{(k-1) \pi}{n}\right)+\sin ^{2}\left(\frac{(l-1) \pi}{n}\right)\right) .
$$

where $1 \leq k, l \leq n$.

We consider the heterogeneous perturbations in the homogeneous space near the $\left(n_{1}, p_{1}\right)$. i.e.,

$$
\begin{aligned}
& \tilde{n}_{(i, j, t)}=n_{(i, j, t)}-n_{1}, \\
& \tilde{p}_{(i, j, t)}=p_{(i, j, t)}-p_{1} .
\end{aligned}
$$

where $\tilde{n}_{(i, j, t)}$ and $\tilde{p}_{(i, j, t)}$ are the perturbations on the density of prey and predator in $(i, j)$ at $t$ th iteration, and noticing that

$$
\begin{aligned}
& \nabla_{d}^{2} n_{(i, j, t)}=\nabla_{d}^{2} \tilde{n}_{(i, j, t)}, \\
& \nabla_{d}^{2} p_{(i, j, t)}=\nabla_{d}^{2} \tilde{p}_{(i, j, t)} .
\end{aligned}
$$

Substituting (35) into (6) and (7), we get

$$
\begin{aligned}
\tilde{n}_{(i, j, t+1)}= & a_{11}\left(\tilde{n}_{(i, j, t)}+\frac{\tau}{h^{2}} d_{11} \nabla_{d}^{2} \tilde{n}_{(i, j, t)}+\frac{\tau}{h^{2}} d_{12} \nabla_{d}^{2} \tilde{p}_{(i, j, t)}\right)+ \\
& a_{12}\left(\tilde{p}_{(i, j, t)}+\frac{\tau}{h^{2}} d_{21} \nabla_{d}^{2} \tilde{n}_{(i, j, t)}+\frac{\tau}{h^{2}} d_{22} \nabla_{d}^{2} \tilde{p}_{(i, j, t)}\right)+O\left(\left(\left|\tilde{n}_{(i, j, t)}\right|+\left|\tilde{p}_{(i, j, t)}\right|\right)^{2}\right), \\
\tilde{p}_{(i, j, t+1)}= & a_{21}\left(\tilde{n}_{(i, j, t)}+\frac{\tau}{h^{2}} d_{11} \nabla_{d}^{2} \tilde{n}_{(i, j, t)}+\frac{\tau}{h^{2}} d_{12} \nabla_{d}^{2} \tilde{p}_{(i, j, t)}\right)+ \\
& a_{22}\left(\tilde{p}_{(i, j, t)}+\frac{\tau}{h^{2}} d_{21} \nabla_{d}^{2} \tilde{n}_{(i, j, t)}+\frac{\tau}{h^{2}} d_{22} \nabla_{d}^{2} \tilde{p}_{(i, j, t)}\right)+O\left(\left(\tilde{n}_{(i, j, t)}|+| \tilde{p}_{(i, j, t)} \mid\right)^{2}\right) .
\end{aligned}
$$


The $O\left(\left(\left|\tilde{n}_{(i, j, t)}\right|+\left|\tilde{p}_{(i, j, t)}\right|\right)^{2}\right)$ can be neglected when the perturbation is very small,. By using the eigenvalues $X_{k l}^{i j}$, multiplied by the above formula, we can obtain

$$
\begin{aligned}
X_{k l}^{i j} \tilde{n}_{(i, j, t+1)} & =a_{11} X_{k l}^{i j} \tilde{n}_{(i, j, t)}+a_{12} X_{k l}^{i j} \tilde{p}_{(i, j, t)}+\frac{\tau}{h^{2}}\left(a_{11} d_{11}+a_{12} d_{21}\right) X_{k l}^{i j} \nabla_{d}^{2} \tilde{n}_{(i, j, t)} \\
& +\frac{\tau}{h^{2}}\left(a_{11} d_{12}+a_{12} d_{22}\right) X_{k l}^{i j} \nabla_{d}^{2} \tilde{p}_{(i, j, t)}, \\
X_{k l}^{i j} \tilde{p}_{(i, j, t+1)} & =a_{21} X_{k l}^{i j} \tilde{n}_{(i, j, t)}+a_{22} X_{k l}^{i j} \tilde{p}_{(i, j, t)}+\frac{\tau}{h^{2}}\left(a_{21} d_{11}+a_{22} d_{21}\right) X_{k l}^{i j} \nabla_{d}^{2} \tilde{n}_{(i, j, t)} \\
& +\frac{\tau}{h^{2}}\left(a_{21} d_{12}+a_{22} d_{22}\right) X_{k l}^{i j} \nabla_{d}^{2} \tilde{p}_{(i, j, t)} .
\end{aligned}
$$

For all $i$ and $j$ obtain

$$
\begin{aligned}
\sum_{i, j=1}^{n} X_{k l}^{i j} \tilde{n}_{(i, j, t+1)}= & a_{11} \sum_{i, j=1}^{n} X_{k l}^{i j} \tilde{n}_{(i, j, t)}+a_{12} \sum_{i, j=1}^{n} X_{k l}^{i j} \tilde{p}_{(i, j, t)}+\frac{\tau}{h^{2}}\left(a_{11} d_{11}+a_{12} d_{21}\right) \sum_{i, j=1}^{n} X_{k l}^{i j} \nabla_{d}^{2} \tilde{n}_{(i, j, t)} \\
& +\frac{\tau}{h^{2}}\left(a_{11} d_{12}+a_{12} d_{22}\right) \sum_{i, j=1}^{n} X_{k l}^{i j} \nabla_{d}^{2} \tilde{p}_{(i, j, t)}, \\
\sum_{i, j=1}^{n} X_{k l}^{i j} \tilde{p}_{(i, j, t+1)}= & a_{21} \sum_{i, j=1}^{n} X_{k l}^{i j} \tilde{n}_{(i, j, t)}+a_{22} \sum_{i, j=1}^{n} X_{k l}^{i j} \tilde{p}_{(i, j, t)}+\frac{\tau}{h^{2}}\left(a_{21} d_{11}+a_{22} d_{21}\right) \sum_{i, j=1}^{n} X_{k l}^{i j} \nabla_{d}^{2} \tilde{n}_{(i, j, t)} \\
& +\frac{\tau}{h^{2}}\left(a_{21} d_{12}+a_{22} d_{22}\right) \sum_{i, j=1}^{n} X_{k l}^{i j} \nabla_{d}^{2} \tilde{p}_{(i, j, t)} .
\end{aligned}
$$

Introduce $\bar{n}_{t}=\sum X_{k l}^{i j} \tilde{n}_{(i, j, t)}$ and $\bar{p}_{t}=\sum X_{k l}^{i j} \tilde{p}_{(i, j, t)}$, then the above is changed into the following form

$$
\begin{aligned}
& \bar{n}_{t+1}=\left(a_{11}-\frac{\tau}{h^{2}}\left(a_{11} d_{11}+a_{12} d_{21}\right) \lambda_{k l}\right) \bar{n}_{t}+\left(a_{12}-\frac{\tau}{h^{2}}\left(a_{11} d_{12}+a_{12} d_{22}\right) \lambda_{k l}\right) \bar{p}_{t}, \\
& \bar{p}_{t+1}=\left(a_{21}-\frac{\tau}{h^{2}}\left(a_{21} d_{11}+a_{22} d_{21}\right) \lambda_{k l}\right) \bar{n}_{t}+\left(a_{22}-\frac{\tau}{h^{2}}\left(a_{21} d_{12}+a_{22} d_{22}\right) \lambda_{k l}\right) \bar{p}_{t} .
\end{aligned}
$$

Calculating the two eigenvalues of the Jacobi matrix of the above equation

$$
\lambda_{ \pm}(k, l)=\frac{1}{2}\left(A_{11}+A_{22}\right) \pm \frac{1}{2} \sqrt{\left(A_{11}-A_{22}\right)^{2}+4 A_{12} A_{21}} .
$$

where

$$
\begin{aligned}
& A_{11}=a_{11}-\frac{\tau}{h^{2}}\left(a_{11} d_{11}+a_{12} d_{21}\right) \lambda_{k l}, A_{12}=a_{12}-\frac{\tau}{h^{2}}\left(a_{11} d_{12}+a_{12} d_{22}\right) \lambda_{k l}, \\
& A_{21}=a_{21}-\frac{\tau}{h^{2}}\left(a_{21} d_{11}+a_{22} d_{21}\right) \lambda_{k l}, A_{22}=a_{22}-\frac{\tau}{h^{2}}\left(a_{21} d_{12}+a_{22} d_{22}\right) \lambda_{k l} .
\end{aligned}
$$

Whether $\left|\lambda_{+}(k, l)\right|>1$ or $\left|\lambda_{-}(k, l)\right|>1$ exists, there will be at least one set of $k$ and $l$ that meets $\lambda_{k l} \neq 0$, and then (40) will not converge. The divergence of (38) indicates the phenomenon of Turing instability.
Next, define

$$
\begin{gathered}
Z(k, l)=\max \operatorname{imum}\left(\left|\lambda_{ \pm}(k, l)\right|\right) . \\
Z_{m}=\max _{k=1}^{n} \max _{l=1}^{n} Z(k, l) .
\end{gathered}
$$

Then, Turing instability occurs. Therefore, (44) is the criterion for judging Turing instability.

\section{NUMERICAL SIMULATIONS}

In this section, we use numerical simulation to illustrate the theoretical results, we select a set of parameters to verify the parameter conditions of Hopf bifurcation and Turing instability. Parameters are fixed on $\delta=0.8, \alpha=5, \beta=0.2, h=2$.

The positive equilibrium point $\left(n_{1}, p_{1}\right)=(0.8077,4.0385)$. We substitute the above parameter into the parameter conditions for the existence of Hopf bifurcation and Turing instability in Section III. The results show that all parameter conditions are satified.

We take $\tau$ and $d_{11}, d_{12}, d_{21}, d_{22}$ of parameters as variables, then can get the bifurcation diagrams, as shown in Figure 1.
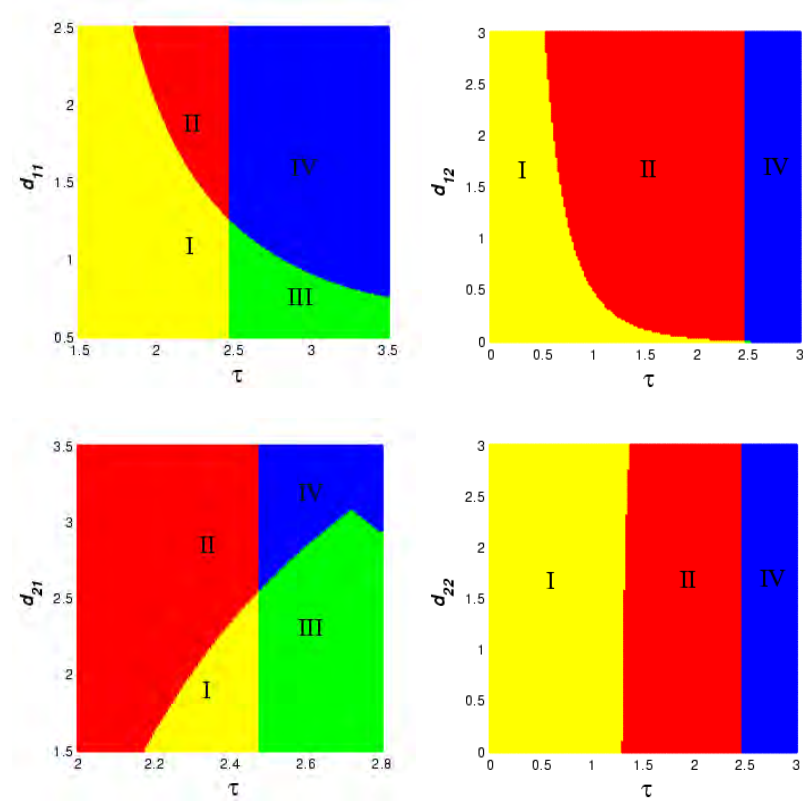

FIGURE I. REGIONS DIAGRAMS SHOWN IN PARAMETER SPACES.

Figure 1 shows four cases of the discrete predator-prey system. Region I is the case of stable state without Hopf bifurcation and Turing instability; region II is the case of pure Turing instability; region III is the case of pure Hopf bifurcation; region IV is the case where both Hopf bifurcation and Turing instability exist. So, we know that III and IV are regions where Turing is instable. 
Figure 2 shows that the predator-prey system will generate the special pattern formation when parameters lie in the region III and IV. Next, because the situation of predator and prey is similar, we only analysis the change of prey patterns. We select the values of parameters

$$
\delta=0.8, \alpha=5, \beta=0.2, h=2, d_{11}=5, d_{12}=10, d_{21}=0.1, d_{22}=0.5 \text {. }
$$
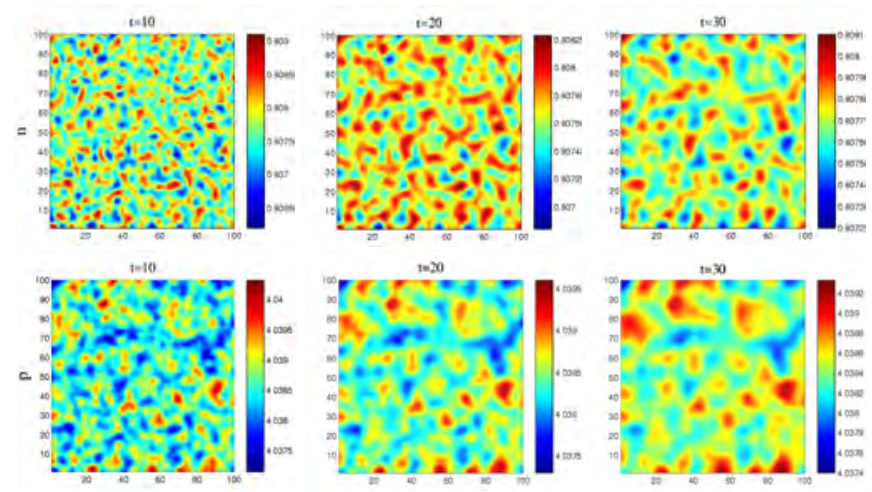

FIGURE II. SPATIAL PATTERNS OF PREY AND PREDATOR.

\section{CONCLUSION}

Hopf bifurcation and Turing instability of a discrete spacetime Holling-Tanner model is investigated in this paper. Previously some research works have been done, where continuous reaction-diffusion models are applied. In this investigate, the research of this model has been developed.

By using discrete system bifurcation theory, it has been proven that the discrete model exists Hopf bifurcation and the parameter conditions of Hopf bifurcation are obtained. According to the two conditions of Turing instability, it has been proved that the system exists Turing instability, and we give the parameter conditions of Turing instability. Finally, numerical simulations are given to verify the theoretical results.

\section{ACKNOWLEDGMENT}

This work is jointly supported by 2015 National traditional Medicine Clinical Research Base Business Construction Special Topics (JDZX2015299) and the Fundamental Research Funds for the Central University FRF-BR-16-019A.

\section{REFERENCES}

[1] Canrong Tian, ZhiLing, ZhiguiLin, Turing pattern formation in a predator-prey-mutualist system, J. Nonlinear Analysis: Real World Applications. 12 (2011) 3224-3237.

[2] Tousheng Huang, Huayong Zhang, Honhju Yang, Ning Wang, Feifan Zhang, Complex patterns in a space- and time-discrete predator-prey model with Beddington-DeAngelis functional response, Commun Nonlinear Sci Numer Simulat 43 (2017) 182-199.

[3] Caiyun Wang, Suying Qi, Spatial dynamics of a predator-prey system with cross diffusion. Chaos, Solitons and Fractals 107 (2018) 55C60.
[4] G. Gambino, M.C. Lombardo, M. Sammartino, Velocity-diffusion method for a Lotka-Volterra system with nonlinear cross anf selfdiffusion, Appl.Numer.Math.59 (2009) 1059-1074.

[5] Santu Ghorai, Swarup Poria. Pattern formation and contral of spatiotemporal chaos in a reaction diffusion prey-predator system supplying. Chaos, Solitions and Fractals. 85 (2016) 57-67.

[6] A. Turing, The chemical basis of morphogenesis, Philos. Trans. Royal. Soc. B 237 (1952) 37C72.

[7] Rodrigues LAD, Mistro DC, Petrovskii S. Pattern formation in a spaceand time-discrete predator-prey system with a strong Allee effect. Theor Ecol 2012;5:341-62.

[8] Domokos G,Scheuring I, Discrete and continuous state population models in a noisy world, J Theor Biol 2004;227:535-45.

[9] James T. Tanner, The stability and the intrinsic growth rates of prey and predator populations, Ecology, Vol. 56, No. 4 (Jul., 1975), pp. 855-867.

[10] Peter A. Braza, The bifurcation structure of the Holling-Tanner model predator-prey interactions using two-timing, SIAM J. Appl. Math. 63 (2003) 889-904.

[11] Malay Banerjee, Santo Banerjee, Turing instabilities and spatio-temporal chaos in ratiodependent Holling-Tanner model, Mathematical Biosciences 236 (2012) 64-76.

[12] A.H. Nayfeh, B. Balachandran, Applied nonlinear dynamics: analytical, computational, and experimental methods. Wiley Interscience; 1995. 